\title{
FIPPS: Fission Product Prompt Gamma- Ray Spectrometer, a New High Efficiency HPGe Array for Nuclear Spectroscopy
}

\author{
P. Mutti ${ }^{1{ }^{*}}$, A. Blanc ${ }^{1}$, M. Jentschel ${ }^{1}$, U. Köster ${ }^{1}$, C. Michelagnoli ${ }^{1}$, E. Ruiz-Martinez ${ }^{1}$ \\ ${ }^{1}$ ILL, Institut Laue-Langevin, BP 156, 6 rue Jules Horowitz, 38042 Grenoble Cedex 9, France \\ *Corresponding author-mutti@ill.eu
}

Within the EXILL campaign a large and efficient cluster of Ge-detectors was installed around a very well collimated neutron beam. This has allowed carrying out rather complete spectroscopic studies close to the line of stability using the (n, gamma) reaction. Neutron rich isotopes were produced by neutron-induced fission on both ${ }^{235} \mathrm{U}$ and ${ }^{241} \mathrm{Pu}$ samples and prompt gamma spectroscopy was carried out. Isotope identification was based on triple gamma coincidences a partially known level scheme and the use of triple coincidences. Because of the high multiplicity coincidence, the sensitivity in the case of weak production yields was substantially reduced.

The EXILL campaign demonstrated the potential physical output from $(n, \gamma)$ and $(n, f)$ reactions and the interest of the nuclear structure community in this type of experiments. Despite the undoubted success of EXILL we learned, as well, that there was space for further improvements on the experimental setup. One of the major problem arising from the analysis of the $(n, f)$ data is the lack of a clear identification of the fission events. This information is very important if one wants to study the less populated nuclei in the fission fragment distribution as well as the kinematic of the fission process itself and therefore determine the mass and the kinetic energy of the fission fragments. The possibility of an additional mass information would improve significantly the capability of the germanium detectors array to identify new $\gamma$-rays. From all these considerations, we decided to propose the construction of a new ILL instrument combining an EXILL-like germanium array with the mass resolving power of a gas filled magnet. FIPPS, Flssion Product Prompt gamma-ray Spectrometer, is now part of the ILL Endurance program phase one, the next ILL's instruments upgrade plan which started this year and will run until 2019. FIPPS initial setup will be very similar to the one of EXILL. 8 high efficiency germanium clover detectors have been purchased this year to form the central ring of the spherical detectors array. The remaining space 
around the target will be filled with additional detectors depending on the specific setup required for the experiments. Therefore, not only more segmented germanium detectors are foreseen to complete the setup but also BEGe (broad energy germanium) that could significantly improve the resolution at very low energy to identify very weak low energy transitions. From the FATIMA collaboration, a set of $\operatorname{LaBr}_{3}(\mathrm{Ce})$ scintillators for picosecond timing studies is also expected to be installed as well as a set of neutron detectors for the study of the fission process. FIPPS has been located at the end position of $\mathrm{H} 22$ thermal neutron guide. The construction of the instrument and its commissioning was completed mid-December last year and FIPPS is now officially operational for users' experiments since the first reactor cycle in 2017. In the last proposals' round of November 2016, assigning beam time only for the first reactor cycle 2017, 8 experiments have been submitted for FIPPS for a total of 108 days of beam time, meaning more than a factor of 2 overbooking; a clear indication of the interest of the nuclear physics community. For fission studies measurements, FIPPS will use a scintillating sample, where the fissioning isotope (e.g. $\left.{ }^{235} \mathrm{U}\right)$ will be dissolved inside scintillator material. This trigger on fission events will clear the gamma spectrum from the huge beta background, reducing, in such a way, the need for high multiplicity coincidence. In a later stage, the HPGe array will be coupled with a gas filled magnet and auxiliary detectors. The presence of the gas filled magnet will dramatically improve the fission tagging allowing the identification of fission products directly. Moreover, the gas filled magnet will be coupled with an ionization chamber to measure the total kinetic energy of the fission fragments allowing detailed fission yield studies. The presentation will briefly discuss the physics case focusing on the experimental results obtained from the first measurement campaign. Further options like a low energy detector cluster, a plunger, a conversion electron spectrometer or neutron detectors and potentially possible experiments will be also discussed. 